\title{
Abstract Feelings Emerging from Haptic Stimulation
}

\author{
Kohske Takahashi ${ }^{1}$, Hideo Mitsuhashi ${ }^{2}$, Kazuhito Murata $^{2}$, \\ Shin Norieda ${ }^{2}$ and Katsumi Watanabe ${ }^{3}$ \\ ${ }^{1}$ Research Center for Advanced Science and Technology, The University of Tokyo \\ Japan Society for the Promotion of Science \\ ${ }^{2}$ NEC System Jisso Research Labs. \\ ${ }^{3}$ Research Center for Advanced Science and Technology, The University of Tokyo \\ Japan Science and Technology Agency \\ Japan
}

\section{Introduction}

Haptic stimulation yields diverse sensations such as pressure, softness and hardness, vibration, and roughness of surface (Katz, 1989). These sensations originate from the sensory process through mechanoreceptors on the skin, such as Pacinian corpuscles, Meissner's corpuscles, Merkel's discs, and Ruffini corpuscles (Fig. 1.). The mechanoreceptors work interactively to build up haptics as an elaborate sensory system. Thus far, a number of studies have investigated the characteristics of the mechanoreceptors, yet the mechanisms have not been fully understood. Instead, these studies have exposed the complexity of the haptic sensory process.

Apart from the mechanoreceptors and the sensory system, cognitive scientists have investigated perceptual processing for haptic stimulation. They have revealed how haptic

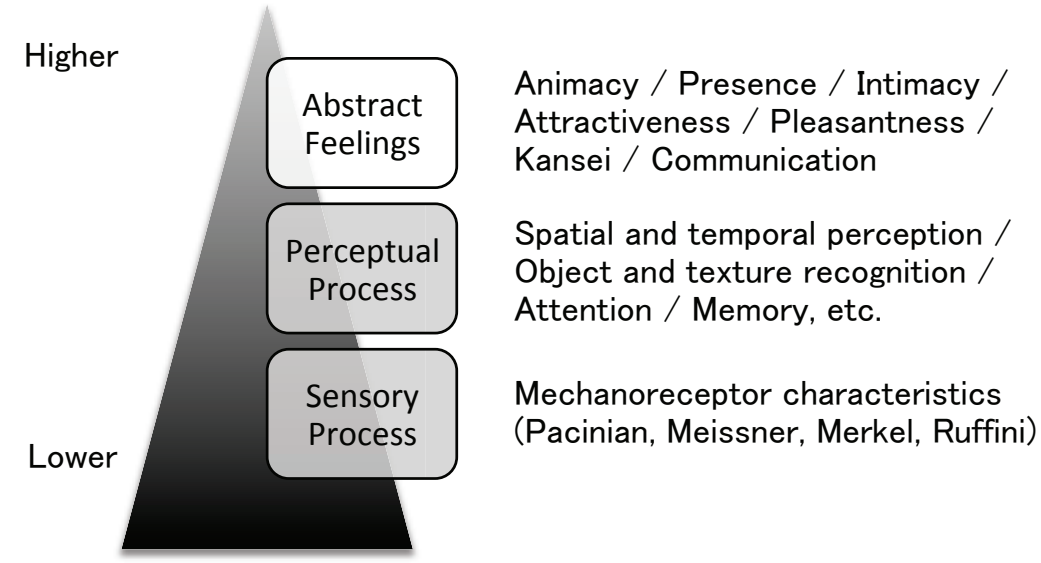

Fig. 1. Schematic illustration of 3 levels of haptic information process. 
inputs are processed in the perceptual system for spatial and temporal perception, object and texture recognition, attention, and memory (Fig. 1.). Here again, haptic perception was revealed to be complex since the haptic perception is built on interaction with other cognitive functions such as action (e.g., active touch) (Gibson, 1962), and other sensory modalities (e.g., proprioceptive, vision and audition).

In addition to these sensory and perceptual systems, and although this aspect is often downplayed, we experience abstract feelings from haptic stimulation, such as feelings of animacy, pleasantness, presence, and intimacy (Fig. 1.). For example, a fisherman can sense the movement of an animate object on his rod when a fish is caught on the hook without seeing or hearing the fish. When we hold hands with a lover, we sense her or his presence through the hands and develop a feeling of pleasure. Hence, there is no doubt that haptic stimulation and these abstract feelings (i.e., feelings of animacy, pleasantness, or presence) are strongly related (Knapp \& Hall, 1992; Morris, 1971; Richmond et al., 2007). Since the haptic system has no receptor that is directly associated with animacy or pleasantness, however, these abstract feelings would emerge from dynamic spatial-temporal patterns of haptic stimulation to the mechanoreceptors and from the perceptual process thereof.

In daily experience, the abstract feelings emerging from haptic stimulation seem not to be trivial. Rather, most haptic experience seems to involve these kinds of abstract feelings to some degree. In fact, haptic sensation is one of the essential channels of interpersonal communication (Richmond et al., 2007). When we communicate with others in daily life, physical contact involving haptic sensation is the most primitive communication channel, as may be widely observed in human infants and animals (Knapp \& Hall, 1992; Richmond et al., 2007). Certainly, the purpose of haptic communication is not to inspect, for example, the stiffness or texture of another person's skin. The purpose of haptic communication would be to feel animacy or the presence of the other and to develop a feeling of pleasure.

The abstract feelings emerging from haptic stimulation are not limited to situations of interpersonal communication. As in the case of the fisherman, we sometimes feel strong and vivid animacy from haptic stimulation. Although the vivid animacy of haptic stimulation would originate from the sensory and perceptual system, we are not usually conscious of the perceptual aspects of haptic stimulation (e.g., stiffness, softness, and frequency). Rather, in most cases we cannot explicitly describe the perceptual aspects of haptic stimulation that lead to animacy. We directly feel the animacy of the object. In other words, such abstract feelings seem to be dominant in our consciousness.

Although the abstract feelings emerging from haptic stimulation can be widely seen and, hence, would be worthwhile to examine empirically, our knowledge thereof is very poor. What types of haptic stimulation yield which kinds of abstract feelings? How do such abstract feelings affect our experience, for example, in interpersonal communication? Do abstract feelings emerging from haptic stimulation interact with the process in the sensory modalities other than with regard to haptics? All these questions are still open.

In this chapter, we review 3 psychological studies recently conducted in our laboratory. All these studies were aimed at understanding how abstract feelings emerge from haptics sensation and, at the same time, how such feelings affect our behavior and experience. The first study investigated the characteristics of haptic stimulation associated with abstract feelings, especially focusing on the animacy of haptic stimulation. The second study investigated the effect of abstract feelings on experience and behavior in interpersonal haptic communication. The third study investigated haptic modulation on abstract feeling in the sensory modalities other than haptics. 
Although we developed a simple haptic display (Fig. 2.) that is suitable for examining abstract feeling emerging from haptic stimulation, the purpose of these studies was not to develop a haptic device to enable users to experience abstract feelings. Instead, we aimed at understanding the human cognitive process in relation to the abstract feelings emerging from haptic stimulation and their impact on human behavior and experience, and also providing useful and basic insights to design a user-friendly haptic device that is able to present abstract feelings efficiently.

\section{Feelings of animacy for vibratory haptic stimulation}

Humans can perceive animacy even from inanimate visual objects. This feeling of the animacy of inanimate objects has been well investigated in the context of animacy perception (Blakemore et al., 2003; Gao et al., 2010; Gao \& Scholl, 2011; Heider \& Simmel, 1944; Scholl \& Tremoulet, 2000). In animacy perception, given the mutually interactive motion of multiple visual objects, human observers sense intention or sociality in artificial visual objects (Fukuda \& Ueda, 2010; Gao et al., 2009; Santos et al., 2008). Furthermore, recent studies have reported that a particular motion pattern of a single visual object could induce animacy perception (Tremoulet \& Feldman, 2000), which implies that apparently higher cognitive appreciation of complex properties such as animacy may originate from low-level sensory processes. Along with these studies, cognitive neuroscientific studies using functional magnetic resonance imaging (fMRI) have shown that the neural activities in primary visual areas, as well as in higher brain areas that are related to the cognitive function (Santos et al., 2010), such as the mirror system (Rizzolatti, 2005) or the theory of mind (Gallese \& Goldman, 1998), might be related to animacy perception (Morito et al., 2009). Thus, the emergence of animacy perception of inanimate objects may originate not only from the top-down cognitive processes of intention or sociality but also partially from the bottom-up processes of primitive sensations such as vibration, softness, and warmth.

As in the fisherman's case, haptic stimulation that induces feelings of animacy in daily life is composed of dynamic and complex patterns. However, given the possibility that primitive sensations might induce feelings of animacy in a bottom-up manner, one challenging question concerns what the lower limit of haptic stimulation is that induces feelings of animacy. To answer this question, Takahashi et al. (2011a) developed a haptic display device and investigated whether simple and cyclic haptic vibratory stimulation can induce feelings of animacy and pleasantness, and if so, what would determine the strength of these feelings.

\subsection{Haptic device}

In order to investigate the abstract feelings for haptic sensation, we developed a haptic display device that is suitable for presenting a soft haptic sensation (Takahashi et al., 2011a) (Fig. 2.). The device can present only vibratory pressure stimulation. However, since the pressure was determined by impressed voltage, and because the impressed voltage was programmable, the device could present arbitrary pressure changes.

The device was composed of an operator personal computer, an amplifier, and a stimulator. The amplifier was attached to a digital-to-analog converter connected to the operator PC. The stimulator comprised (a) an actuator, (b) a medium, (c) a chassis, and (d) a contact plate (Fig. 2B.). The actuator can be one that changes in size by impressed voltage. Any incompressible fluid may work as the medium. The chassis must be a rigid body, be filled with liquid, and be completely enclosed. We chose a piezoelectric vibrator for the actuator (diameter was $54.5 \mathrm{~mm}$ ), water for the medium, an acrylic resin and polyurethane-tube (inner and outer 
A

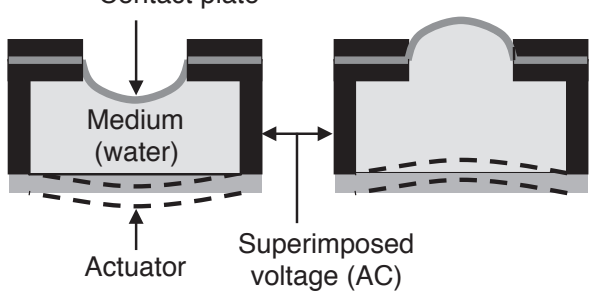

B

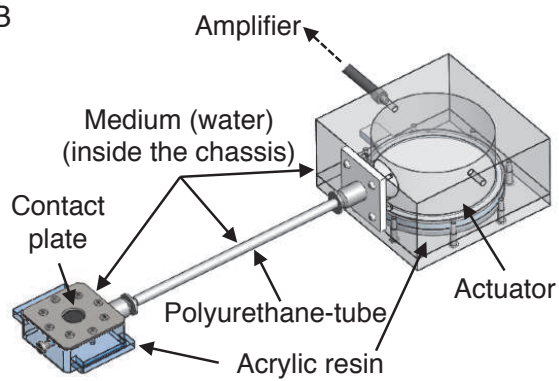

Fig. 2. Haptic device. A. Working principle of the device. B. Appearance and structure of the haptic stimulator used in the experiment. The figures were modified from Takahashi et al. (2011a).

diameters were $4 \mathrm{~mm}$ and $6 \mathrm{~mm}$, respectively) for the chassis, and silicon rubber for the contact plate (the diameter was $16 \mathrm{~mm}$, and the size of the box, including contact plate, was $30 \mathrm{~mm} \times 30 \mathrm{~mm} \times 12 \mathrm{~mm}$ ).

The working principle of the stimulator was as follows (Fig. 2.). The waveform of pressure change was defined using the operator PC and sent to the stimulator through the DA converter and the amplifier. The input terminal of the actuator of the stimulator was attached to the output terminal of the amplifier. After a start signal was sent to the DA converter, actual voltage was conveyed to the stimulator through the amplifier, which induced the vibration of the actuator. The actuator and contact plate parts were connected with a polyurethane-tube, which was also water-filled. The vibration of the actuator was conveyed to the contact plate through the medium, leading to the production of haptic stimulation at the contact plate.

A significant feature of the device we developed is as follows:

\section{Flexibility of the material of the contact plate}

Although we chose silicon rubber for the contact plate because we wanted to create a limp sensation, one can choose a hard or textured material (e.g., harsh, scratchy) as required.

\section{Flexibility of the placement of the contact plate}

In our device, the actuator and contact plate were not solidly attached but were connected by a flexible material (i.e., the polyurethane-tube), and the component, including the contact plate, was small and lightweight. The mobility of the components enables the stimulation of any part of the body surface, and hence enables one to compare the haptic stimulation among the different body parts using identical stimuli.

\section{Dynamic range of pressure displacement}

The device can present a wide range of pressure displacement. The ratio of the size of the actuator and contact plate determines the amplitude of the displacement of the contact plate; a smaller contact plate and a larger actuator lead to a larger amplitude. The larger amplitudes of the displacement lead to a larger pressure change in the haptic sensation.

\section{Programmable pressure change}

Since the pressure change is defined as the change of impressed voltage and because the impressed voltage is fully programmable, the device can present any temporal profile of pressure change. 


\subsection{Animacy and pleasantness emerges from haptic sensation}

Using the haptic display device, Takahashi et al. (2011a) conducted a psychological experiment to investigate the effects of the frequency of vibratory stimulation on felt animacy and pleasantness.

Six volunteers participated in the experiment. In each trial, haptic stimuli were presented for $3 \mathrm{~s}$ after a $1 \mathrm{~s}$ blank period, which was followed by an evaluation display on a touch panel screen. The participants were instructed to evaluate the strength of felt animacy and the pleasantness of the haptic stimuli on a 7-point scale.

They conducted two conditions according to the body parts where the haptic stimuli were presented-finger condition and wrist condition. In the finger condition, the haptic stimuli were presented to the pad of the right index finger. The participants were instructed to softly touch the contact plate by their right index finger. In the wrist condition, the haptic stimuli were presented to the inner side of the right wrist. The component, including the contact plate, was fixed to the right wrist using a band. A haptic stimulus was sinusoidal vibratory stimulation with variable frequency. The frequency was randomly chosen from $0.5,1,2,5,10$, 25, and $50 \mathrm{~Hz}$.

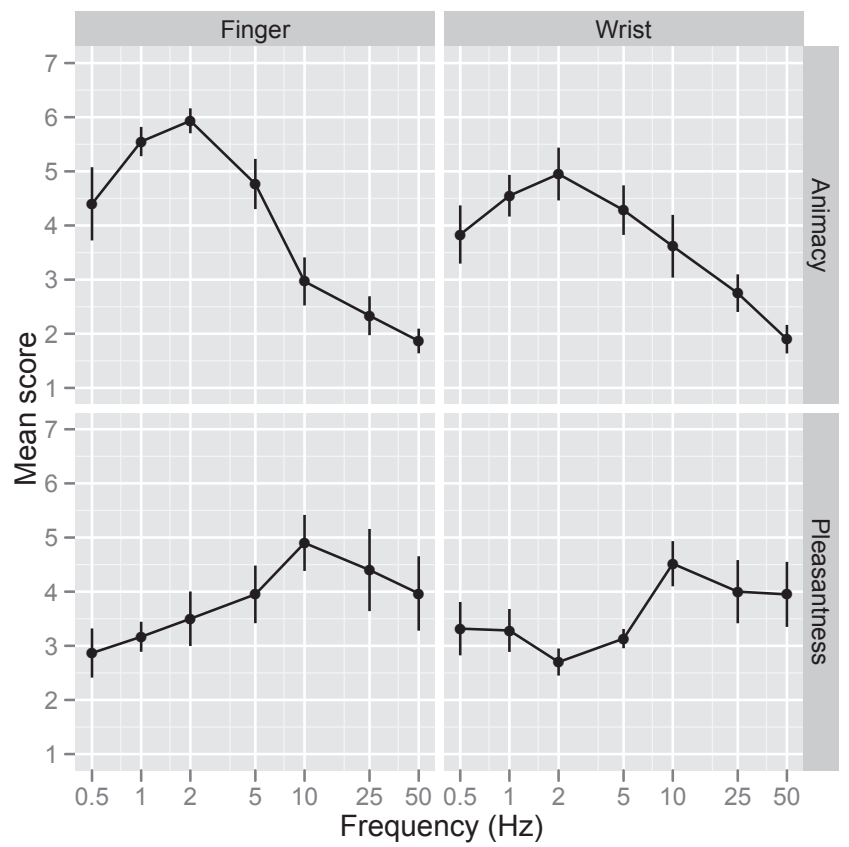

Fig. 3. Mean scores of feelings of animacy (top) and pleasantness (bottom) as a function of stimulus frequency and sensed body part. Error bars indicate the standard errors of means. The figures were modified from Takahashi et al. (2011a).

Fig. 3. shows the felt animacy and pleasantness as a function of the stimulus frequency and body parts. A statistical test (two-way repeated measures ANOVA) revealed that the stimulus frequency significantly affects felt animacy $(\mathrm{F}(6,30)=13.1, \mathrm{p}<.01)$, whereas the body parts did not significantly affect felt animacy $(F(1,5)=2.98, p=.15)$. Thus, the effects of the 
stimulus frequency on animacy perception were qualitatively similar between finger and wrist stimulation, although they were quantitatively different. At first glance, the low-frequency stimulation (from 0.5 to $5 \mathrm{~Hz}$ ) appeared to induce stronger feelings of animacy than the high-frequency stimulation (from 10 to $50 \mathrm{~Hz}$ ) in both the finger and wrist conditions. The peak rating of animacy was around 1 to $2 \mathrm{~Hz}$ in both conditions, but the peak looked more prominent in the finger condition than in the wrist condition. The felt animacy in the finger condition was significantly, or marginally significantly, stronger at $1 \mathrm{~Hz}(\mathrm{~F}(1,5)=7.08, \mathrm{p}<$ $.05)$ and $2 \mathrm{~Hz}(\mathrm{~F}(1,5)=4.27, \mathrm{p}<.10)$ and significantly weaker at $25 \mathrm{~Hz}(\mathrm{~F}(1,5)=7.79, \mathrm{p}<.05)$ than that in the wrist condition. These results imply that the finger was more sensitive to the frequency difference of haptic stimulation than the wrist in feeling animacy. Note that the patterns of frequency dependence were consistent among different participants.

With regard to the feeling of pleasantness, neither the stimulus frequency nor the body parts affected the pleasantness of the haptic stimuli, although a visual inspection of the data would suggest that the high-frequency stimulation induced stronger feelings of pleasantness than the low-frequency stimulation.

Fig. 4. shows the scatter plot of the finger condition vs. wrist condition (top) and that of the animacy rating vs. pleasantness rating (bottom). Each data point indicates the data from individual participants. There were strong correlations between the finger and wrist sensations in both the animacy $(\mathrm{r}=0.83, \mathrm{t}(40)=9.40, \mathrm{p}<.01)$ and pleasantness $(\mathrm{r}=0.71, \mathrm{t}(40)$ $=6.34, \mathrm{p}<.01)$ evaluations. These results supported the theory that feelings of pleasantness and animacy were qualitatively similar between the finger and wrist stimulation.

On the other hand, the feelings of animacy and pleasantness did not correlate in both finger conditions $(\mathrm{r}=-0.13, \mathrm{t}(40)=0.80, \mathrm{p}=.43)$ and wrist $(\mathrm{r}=0.04, \mathrm{t}(40)=0.25, \mathrm{p}=.80)$. However, the pattern of results would not support the theory that the two evaluations were independent. A visual inspection, as well as the Lowess regression of the scatter plot, exhibited an inverse $\mathrm{V}$ shape, especially in the finger condition. These results suggest that the moderate strength of animacy is associated with higher pleasantness.

Thus, the experimental study in Takahashi et al. (2011a) suggested the following:

1. The stimulus frequency systematically affects felt animacy. The lower stimulus frequency around 1-2 Hz induced the strongest feeling of animacy compared with the higher stimulus frequency.

2. The effect of the stimulus frequency on animacy and pleasantness were comparable between different body parts-finger and wrist.

3. The moderate level of animacy of haptic sensation was associated with higher pleasantness.

In another series of studies, Takahashi, Mitsuhashi, Norieda, Murata \& Watanabe (2010) showed that the patterns of frequency dependence were partially different between finger and wrist stimulation. Takahashi, Mitsuhashi, Norieda, Murata \& Watanabe (2010) showed a V-shaped curve for animacy evaluation as a function of the stimulus frequency in the wrist stimulation: the felt animacy was stronger for $1-2 \mathrm{~Hz}$ stimulus frequency, weak for $5-10 \mathrm{~Hz}$ frequency, and stronger again for $25 \mathrm{~Hz}$ frequency. The main difference between Takahashi, Mitsuhashi, Norieda, Murata \& Watanabe (2010) and Takahashi et al. (2011a) was the pressure of water inside the haptic stimulator (Fig. 2A.). In Takahashi et al. (2011a), the chassis was filled with water to the maximum, while this was not the case in Takahashi, Mitsuhashi, Norieda, Murata \& Watanabe (2010). Although further examinations are warranted, this difference in pressure might lead to the different patterns of frequency dependence. Perhaps 

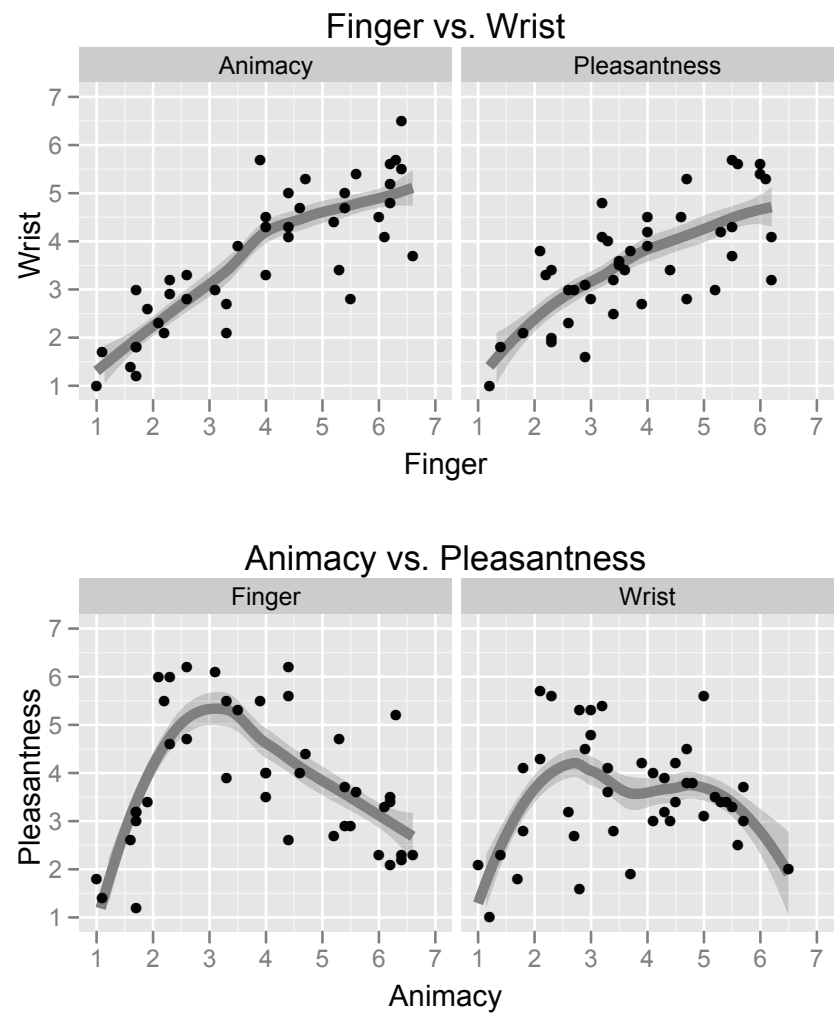

Fig. 4. Top: Scatter plot of finger and wrist sensations of animacy and pleasantness. Bottom: Scatter plot of feeling of animacy and pleasantness in the finger and wrist conditions. In both panels, each point indicates individual participants. The dark gray curve indicates the Lowess (locally weighted scatter plot smoothing) regression curve (degree of smoothing = 0.75 , degree of polynomials $=2$ ). The light gray area indicates the standard error of the Lowess regression. The figures were modified from Takahashi et al. (2011a).

the feeling of animacy for haptic stimulation was susceptible to the pressure as well as to the stimulus frequency.

\subsection{Summary}

Takahashi et al. (2011a) showed that the stimulus frequency strongly influenced the feeling of animacy vibratory stimulation, with the lower frequency $(1-2 \mathrm{~Hz})$ inducing stronger feelings of animacy than the higher frequency $(25-50 \mathrm{~Hz})$. The frequency dependence was consistent among different participants. The animacy for haptic stimulation would partially but intrinsically originate from temporal patterns of haptic stimulation.

How do human observers come to feel animacy from the simple vibratory stimulation of an inanimate object? Two accounts, which are not mutually exclusive, would be possible. First, humans may intrinsically feel animacy from low-frequency haptic stimulation. Second, 
animacy perception may emerge as a result of the frequent experience of touching animate objects such as animals. While the stimulation from these animate objects would involve more complex patterns than those noted in Takahashi et al. (2011a), the frequency of stimulation might be the major factor that induces the feeling of animacy. Therefore, it would be intriguing to survey the frequencies of haptic stimulation from these animate objects in the real world and to compare them to the results of the experimental study in Takahashi et al. (2011a).

\section{Abstract feelings in interpersonal haptic communication}

In social life, nonverbal communication is crucial for conveying abstract feelings (i.e., "Kansei" information), which is too ambiguous and abstract to be described verbally, and for sharing them with others. When we communicate with others in our daily lives, we use various nonverbal channels such as gestures, facial expressions, or gaze. Among them, haptic communication is the most primitive channel, and it is widely seen in human infants and animals (Knapp \& Hall, 1992). In particular, haptic communication plays a dominant role in directly conveying abstract feelings such as emotions (Richmond et al., 2007). Although nonverbal haptic communication has been less understood as compared to verbal communication, some researchers have challenged the kinetic taxonomy of physical contact or categorization based on the meaning of physical contact (Morris, 1971).

The sense of touch or haptics is complex (Katz, 1989), bidirectional, and interactive (e.g., active touch (Gibson, 1962)). Therefore, what is conveyed in interpersonal haptic communication cannot be determined straightforwardly. Instead, the perceptual process for haptic communication itself, the cognitive process for understanding the intention of others, and the context (e.g., the social position of others) would interactively affect what is conveyed. The abstract feelings conveyed in haptic communication are loosely categorized on the basis of the types of motion. For example, stroking someone's head may express affection, and shaking hands may express amity. However, the abstract feeling in haptic communication is also subject to the context of the communication (Richmond et al., 2007). Let us consider a handshake between politicians, that between a mother and a baby, and that between close friends at the time of farewell. It is almost certain that the feelings of the people are not identical in these contexts, although the motions involved in the haptic communication are rather similar.

Given the importance of haptic communication in conveying and sharing abstract feelings, we expect that the quality of experience shared with others could be altered by adding haptic communication. Some innovative studies have already tried to develop haptic devices enabling interpersonal communication and have empirically examined their effect on task performance and subjective impressions (Fujita \& Hashimoto, 1999; Reiner, 2004; Sallnäs et al., 2000; Wang et al., 2004). For example, Fujita \& Hashimoto (1999) showed that users could feel the presence of another person through haptic bidirectional communication even without seeing each other. Sallnäs et al. (2000) also reported that those who were performing collaborative tasks in a virtual environment felt increased reality or presence and showed an improvement in their task performance due to the force feedback from the virtual environment.

In this chapter, we discuss abstract feelings in interpersonal haptic communication. One question concerns how people experience interpersonal haptic communication in their daily activity. Takahashi, Mitsuhashi, Norieda, Sendoda, Murata \& Watanabe (2010) and Takahashi et al. (2011c) conducted a psychological survey to investigate this question. Another question is whether haptic communication could change the subjective quality of an experience, and if 
so, how. To examine this question, Takahashi et al. (2011b) conducted an experimental study investigating the effects of haptic communication on the quality of participants' experiences, as well as on their impression of the person with whom they communicated.

\subsection{Psychological survey on interpersonal haptic communication}

In a series of psychological surveys, Takahashi, Mitsuhashi, Norieda, Sendoda, Murata \& Watanabe (2010) and Takahashi et al. (2011c) investigated how people experience interpersonal haptic communication in their daily activity.

Takahashi, Mitsuhashi, Norieda, Sendoda, Murata \& Watanabe (2010) presented observers with pictures depicting two persons performing either positive (e.g., hugging, shaking hands) or negative (e.g., grappling) haptic communication. The observers were asked to make a subjective evaluation of the depicted communication concerning its desirability, frequency of experience, and impressiveness. All these evaluations had a positive correlation $(R>0.59, \mathrm{p}$ $<.001)$, suggesting that the haptic communication that was more frequently experienced and that made a stronger impression tended to be evaluated as more desirable. Although these correlations cannot reveal a causal relationship, one possibility may be related to the widely known psychological phenomenon of "mere exposure effect" (Zajonc, 1968), and the other is related to "directional forgetting" (Golding \& Macleod, 1998).

The observers were also asked to identify the person who was imagined to be involved in the communication depicted in the picture. The persons associated with the desirable communication were a spouse, a child, a mother, a son or a daughter, a father, and a grandparent. Thus, haptic communications that recall family members are more desirable.

Takahashi et al. (2011c) asked their observers to evaluate two types of haptic communication, tapping and padding, by using adjectives, and examined what factors reside in haptic communication. Table 1 shows the results of the factorial analysis. The results suggested that two factors represent interpersonal haptic communication. One factor affected the evaluation of lightsome, pleasant, comfortable, and warm feelings, and the other factor affected the evaluation of strong, rough, and active feelings. Thus, the first factor was associated with the abstract feelings of haptic communication, while the second factor was relevant to the characteristics of motion. These structures were common between the two types of haptic communications. Furthermore, the first factor strongly affected the desirability of the haptic communications when compared with the second factor, suggesting that the abstract feeling emerging from the communication, not the motion itself, strongly influenced the desirability of the haptic communication.

\begin{tabular}{c|rr|rr}
\hline & \multicolumn{2}{|c|}{ Tapping } & \multicolumn{2}{c}{ Patting } \\
\hline Adjective & Factor 1 & Factor 2 & Factor 1 & Factor 2 \\
\hline Lightsome & 0.87 & 0.01 & 0.80 & 0.07 \\
Pleasant & 0.86 & 0.05 & 0.83 & 0.09 \\
Comfortable & 0.86 & -0.07 & 0.70 & -0.26 \\
Warm & 0.83 & -0.13 & 0.79 & -0.25 \\
Soft & 0.46 & -0.51 & 0.59 & -0.44 \\
Strong & -0.06 & 0.90 & 0.19 & 0.67 \\
Rough & -0.22 & 0.80 & -0.18 & 0.73 \\
Active & 0.47 & 0.61 & 0.69 & 0.35 \\
\hline
\end{tabular}

Table 1. First and second component score of each adjective for describing two types (tapping and patting) of haptic interpersonal communication. Data were from Takahashi et al. (2011c). 
These psychological surveys in Takahashi, Mitsuhashi, Norieda, Sendoda, Murata \& Watanabe (2010) and Takahashi et al. (2011c) suggested that desirable haptic communication is experienced with closer persons in daily life (Knapp \& Hall, 1992; Morris, 1971; Richmond et al., 2007), and that the abstract feelings emerging from the haptic communication are essential for determining the desirability of the communication.

\subsection{Experimental study on shared experience via interpersonal haptic telecommunication}

Takahashi et al. (2011b) conducted an experimental study to investigate how interpersonal haptic communication affects shared experience and found that haptic communication could improve the quality of experience as well as the impression of the other person. They examined the effects of haptic communication on the quality of the experience shared with another person along with the impression of the other person with whom they communicated. In the experiment, two persons watched a movie at the same time. All forms of communication except haptic communication were completely removed. The persons could communicate by triggering a haptic stimulation in the other person.

Forty graduate and undergraduate students participated in the experiment in 20 pairs, which were divided into two groups of 10 pairs each. Each pair in the groups was assigned to one of two experimental conditions. In the experiment, two LCD TVs were placed on two desks. The participants were seated at the desks and simultaneously watched a movie on the TV with headphones. The desks were separated by a partition; hence, the participants could not see or hear each other. Each participant held a push button with his or her left hand and touched the haptic device (Fig. 2.) with his or her right hand. The button sent a signal to the operator PC when it was pressed and released. The haptic stimulator generated 2-Hz of vibratory pressure stimulation when receiving a signal from the operator PC. The operator PC sent a signal to the haptic stimulator of one person when the other person pressed his or her button. The timing of the button press/release was recorded by the operator PC. Takahashi et al. (2011b) chose a Japanese comedy movie clip of approximately $220 \mathrm{~s}$ duration as the visual stimulus. The movie was played on two temporally synchronized screens.

In an experimental session, the participants were instructed to watch the movie and to press the button while they found it hilarious. They were also told explicitly that pressing their button sent a haptic stimulation to the other person and that the other person's button press triggered a haptic stimulation in their sensor. They experienced this haptic communication prior to the experimental session. Thus, the participants were aware that they could perceive the other person's response through the haptic stimulation. The participants were explicitly instructed to decide on their own whether to press the button and to avoid following the lead of the other person.

One of the two experimental groups was an uninterrupted group, in which the button press always triggered the haptic stimulation to the other person throughout the experimental session. The other experimental group was an interrupted group, wherein the haptic stimulation was interrupted for $90 \mathrm{~s}$, between $75 \mathrm{~s}$ and $165 \mathrm{~s}$ after the movie started. The participants in the interruption group were not told of the interruption of the haptic stimulation prior to the experimental session; hence, they believed that the absence of haptic stimulation indicated that the other person did not find the movie hilarious. After watching the movie, the participants answered a questionnaire consisting of 8 questions and using a 7-point scale (Table 2).

Using a post-experiment questionnaire survey, Takahashi et al. (2011b) found that the interruption of haptic communication affected the subjective evaluation of the quality of the 
Q1 1 The movie was hilarious.

Q2 It felt as if the movie was more hilarious due to watching it with the other.

Q3 It seemed as if I wanted to convey the hilarious feeling to the other.

Q4 It seemed as if I could convey the hilarious feeling to the other.

Q5 I was concerned about the other.

Q6 It seemed as if the other's hilarious feeling could be convey to me.

Q7 It felt as if I agreed with the other's hilarious feeling.

Q8 It felt as if I wanted to watch the movie again with the other.

Table 2. Questions on the questionnaire. Each question was rated on a 7-point scale (1: strongly disagree-7: strongly agree).
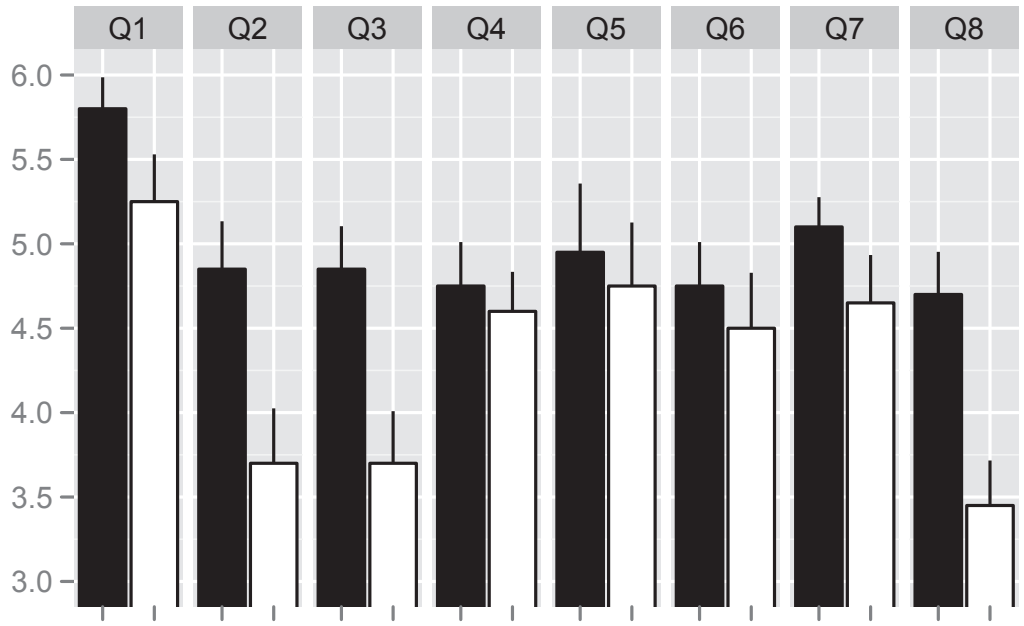

Uninterrupted $\square$ Interrupted

Fig. 5. Mean rating score for each question of the questionnaire. Error bars indicate the standard errors of means. The figure was modified from Takahashi et al. (2011b).

experience and the impression of the other person. Fig. 5 shows the ratings for each question (Table 2). A higher score indicates a stronger agreement with the question. The statistical test (t-test) showed that the rating scores of three questions (Q2, Q3, and Q8) were significantly higher in the uninterrupted group than in the interrupted group (all $t(38)>2.66, \mathrm{p}<.05$ ).

Q2 asked whether the participants felt that the experience with the other person made the movie more hilarious. The participants' impressions may be attributed more to the sharing of the experience with the other person if the haptic communication was uninterrupted. Q3 asked if the participants felt eager to convey their feelings to the other person, and Q8 asked if the participants felt they would like to watch a movie with the other person again in the future. These questions concerned the participants' feelings toward the other person, and the interruption of haptic communication decreased such responses toward the other person.

On the other hand, as for Q1, where the participants were asked to rate the hilariousness of the movie itself, the rating score of the uninterrupted group was slightly higher than that 
of the interrupted group, but the difference was not statistically significant $(\mathrm{t}(38)=1.64, \mathrm{p}=$ 0.11). It seemed that the subjective evaluation of the quality of the experience itself was less susceptible to the absence or presence of haptic communication.

As for the other questions, Q4, Q5, Q6, and Q7, the rating scores were comparable between the two experimental groups. Q4 and Q6 asked whether feelings were conveyed efficiently between the participants during the experiment. Q5 and Q7 asked about their involvement and agreement with the other person during the experiment. These questions were partially similar to Q3 and Q8 in that all the questions were related to the communication with the other person. However, while Q4, Q5, Q6, and Q7 focused on the feelings of the other person during the experiment, Q3 and Q8 referred to the strength of the intentions toward the other person in present and future communication. In summary, the interruption of haptic communication had little effect on the subjective evaluation of the shared experience with others. However, it modulated the impression of the other person, especially the intention to build a relationship with him or her.

The results of an exploratory factor analysis of the subjective evaluation were interesting, since one factor (Factor 1) affected Q2, Q3, and Q8, which showed the group difference in the rating score, and another factor (Factor 2) affected Q6 and Q7 (the loadings of Factor 2 for Q1 and Q4 were also comparatively high). The straightforward interpretation would be that the questions affected by Factor 1 were relevant to the attitude toward the other person, and that the questions affected by Factor 2 were relevant to the feeling of the experience itself. The questions related to Factor 2 did not show any group difference in the rating score. Taken together, the interruption of haptic communication did not degrade the retrospective impression of the experience itself but did reduce the participants' concern toward the other person with whom they communicated.

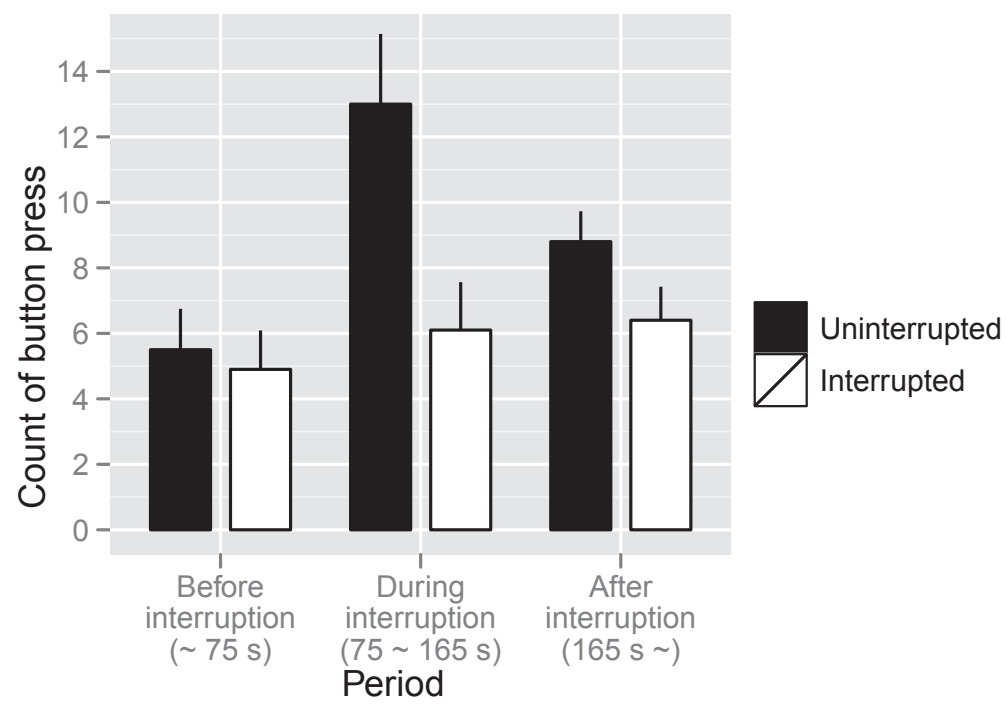

Fig. 6. Number of button presses. Error bars indicate the standard errors of means. The figure was modified from Takahashi et al. (2011b). 
In addition to the subjective evaluation using the post-experiment questionnaire survey, the interruption of haptic communication affected the participants' behavior while watching the movie-namely, the number of button presses during the movie presentation. Fig. 6 . shows the total number of button presses for three different periods. The first one was the period before the interruption of haptic stimulation; hence, there was inherently no difference between the two groups. The numbers of button presses did not differ between the two groups in the first period $(\mathrm{t}(18)=0.62, \mathrm{p}=0.54)$. In the second period, the haptic stimulation was cut off between 75 and $165 \mathrm{~s}$ for the interrupted group. In this period, the number of button presses in the uninterrupted group was significantly larger than that in the interrupted group $(t(18)=2.65, p<0.05)$. The third period was the one after the interruption. Although the number of actions was slightly larger in the uninterrupted group, the difference was not significant $(\mathrm{t}(18)=1.59, \mathrm{p}=0.13)$.

In the questionnaire survey, we found that the retrospective evaluation of how hilarious the movie was did not significantly differ between the two groups (Q1). In contrast, the behavioral performance clearly showed that the number of button presses-namely, how often the participants felt the movie was hilarious-was decreased by the interruption of the haptic communication. These results suggest that the haptic communication implicitly modulated the quality of the experience, although the participants could not be explicitly aware of that.

\subsection{Summary}

Both psychological surveys (Takahashi et al., 2011c; Takahashi, Mitsuhashi, Norieda, Sendoda, Murata \& Watanabe, 2010) and the experimental study (Takahashi et al., 2011b) showed the importance of haptic stimulation in interpersonal communication. The desirability of haptic communication was associated with the factor relevant to abstract feelings (lightsome, pleasant, comfortable, and warm) (Takahashi et al., 2011c; Takahashi, Mitsuhashi, Norieda, Sendoda, Murata \& Watanabe, 2010). The presence of haptic communication improved the quality of experience and the impression of the other person as well (Takahashi et al., 2011b). As the vibratory haptic stimulation could affect the quality of experience, the presence of haptic communication, not the contents of the stimulation, may be essential for influencing the quality of experience. Abstract feelings emerging from the haptic stimulation in interpersonal communication would be important for persons who share a high-quality experience and have a good impression of each other (Morris, 1971).

\section{Cross-modal effect of haptic stimulation in abstract feelings}

Humans have multiple sensory modalities for accessing the external world, such as vision, audition, and, of course, haptics. Although inputs into different sensory modalities are processed in different brain regions at early sensory levels, different sensory modalities are no doubt interactively processed at the perceptual level to build up unified perceptions of the external world, which is referred to as cross-modal interaction (Shams \& Kim, 2010; Shimojo \& Shams, 2001). For example, the ventriloquism effect (Alais \& Burr, 2004) and McGurk effect McGurk \& MacDonald (1976) are well known phenomena demonstrating visual modulation on auditory perception.

Although cross-modal interaction has mainly been examined using vision and audition, cross-modal interaction at the perceptual level is not limited between them. Inputs into haptics can affect perceptual processes in the sensory modalities other than haptics. For example, the dominant interpretation for an ambiguous visual figure (e.g., the Necker cube) 
is biased by haptic inputs to make visual and haptic perception consistent (Blake et al., 2004; Bruno et al., 2007). Furthermore, inputs into haptics and vision are integrated in a statistically optimal fashion (Ernst \& Banks, 2002; Ernst \& Bülthoff, 2004). Thus, a good amount of experimental evidence has shown that inputs into haptic modality can affect perceptual processes in the modalities other than haptics beyond the boundary of sensory modalities.

Consequently, can abstract feelings emerging from different sensory modalities also interact with or modulate from each other? Recent experimental studies suggest that cross-modal interaction takes place at quite high levels of the perceptual process, such as emotion recognition (Bertelson \& de Gelder, 2005; Tanaka et al., 2010). For example, face emotion and voice emotion are strongly related. Emotion recognition of facial expression is biased when the voice expresses emotion different from that of the face, and vice versa (Bertelson \& de Gelder, 2005).

Although it is true that perceptual and emotional processes interact with each other between different sensory modalities, it is still open if abstract feelings such as animacy can be shared among multiple sensory modalities. However, in daily experience, it seems that such abstract feelings simultaneously emerge from multiple sensory modalities. For example, a mother and a baby communicate through eyes, ears, and hands to feel presence or animacy from each other. Therefore, it would be possible to expect that cross-modal interaction also appears for the abstract feelings. In the following section, we introduce an original research recently conducted in our laboratory, which examined if haptic stimulation can modulate abstract feelings for voice stimuli.

\subsection{Voice perception modified by haptic sensation}

In order to investigate if the abstract feeling emerging from haptic sensation can affect the cognitive process of sensory modalities other than haptics, we conducted an experiment wherein observers evaluated abstract feelings for another person's voice. In the experiment, the voice stimuli either were or were not accompanied by haptic stimulation.

Twenty-four paid volunteers took part in the experiment. All of the participants reported normal hearing and haptic sensitivity. They were naive as to the purpose of the experiment. The haptic stimulus was $2 \mathrm{~Hz}$ sinusoidal vibratory stimulation presented by the custom-made haptic display device shown in Fig. 2. Voice stimuli were presented though headphones. The voice stimuli were 5 Japanese greeting phrases from daily conversation ("Ohayou," "Irasshai-mase," "Arigatou-gozaimashita," "Oyasumi-nasai," and "Otsukare-sama-deshita," which correspond to "Good morning," "Can I help you?" "Thank you," "Good night," and "That's wrap," respectively). The voices were spoken by 5 professional voice actors and were emotionally-neutral.

In a trial, the participants touched the haptic device with their right index fingers. After the participants pressed the space bar of an operator PC, the voice stimulus was presented. In half of the trials, the voice stimuli were accompanied by the haptic vibratory stimulation. The haptic stimulus was presented for $3 \mathrm{~s}$ and the voice stimuli were presented during the haptic stimulation. In the other half of the trials, the haptic stimulation was not presented and only the voice stimuli were presented.

After the stimulus presentation, the participants were asked to rate how strongly the voice yielded various abstract feelings-animate, pleasant, warm, familiar, or attractive-on a 7 -point scale. They were explicitly told to ignore the contents of the conversation as well as the haptic stimulation. One practical trial was followed by two trials with haptic stimulus 
and two trials without haptic stimulus. The order of trials and the contents of the conversation were randomized.

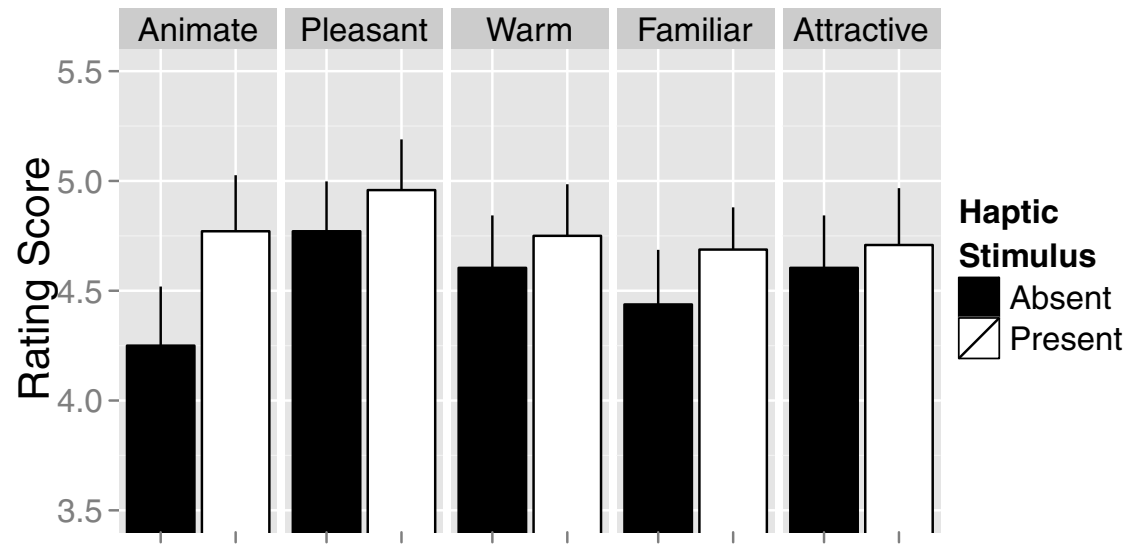

Fig. 7. Rating scores of abstract feelings for voice with and without haptic stimulus. The error bars indicate standard errors of means.

Fig. 7. shows the mean rating scores of 5 questions. The feeling of animacy was significantly stronger when the voice was accompanied by the haptic stimulus than when the voice was presented alone (one-tailed paired t-test, $\mathrm{t}(23)=1.87, \mathrm{p}<.05$ ). As for the other questions, although the means rating score was slightly higher when the haptic stimulus was present, the difference between the haptic-present and the haptic-absent trial was not statistically significant (Pleasant: $\mathrm{t}(23)=0.85, \mathrm{p}=.20$, Warm: $\mathrm{t}(23)=0.50, \mathrm{p}=.31$, Familiar: $\mathrm{t}(23)=0.82, \mathrm{p}$ $=.21$, Attractive: $\mathrm{t}(23)=0.33, \mathrm{p}=.37$ ).

Thus, the results of the experiment clearly showed that the feeling of voice animacy increased when haptic vibratory stimulation was added to the voice stimuli. Even when the haptic stimulation was task irrelevant and the observers were explicitly told to ignore it, the haptic stimulation modulated the abstract feelings for voice stimuli. As described in Section 2, Takahashi et al. (2011a) and Takahashi, Mitsuhashi, Norieda, Murata \& Watanabe (2010) showed that a strong feeling of animacy emerged from $1-2 \mathrm{~Hz}$ of haptic vibratory stimulation. The fact that only the feeling of animacy, that was expected to emerge from haptic stimulatin, was manifest, out of 5 abstract feelings, seems to demonstrate that the effect was not due to general bias in rating higher when the voice was accompanied by haptic stimulus.

\subsection{Summary}

We investigated if haptic stimulation can modulate abstract feelings for voice stimuli. The results showed that haptic stimulation that is associated with feelings of animacy can increase the felt animacy for voice stimuli. Perhaps the abstract feeling emerging from haptic stimulation interacts with inputs into sensory modalities other than haptics.

These results suggested that the processes concerning abstract feelings, as well as perceptual (Shams \& Kim, 2010; Shimojo \& Shams, 2001) and emotional (Bertelson \& de Gelder, 2005; Tanaka et al., 2010) processes, work interactively among different sensory modalities. It may be plausible to argue that higher levels of cognitive processes have vaguer boundaries between different sensory modalities (Fig. 1.). 


\section{Overall summary and future direction}

In this chapter, we introduced a series of psychological studies recently conducted in our laboratory and discussed the abstract feelings emerging from haptic stimulation. These studies aimed at investigating how humans conceive abstract feelings such as animacy, presence, or pleasantness from simple haptic stimulation and how such abstract feelings affect human experience and behavior.

In sum, we showed that haptic stimulation and abstract feelings are closely related. The first study showed that a feeling of animacy emerges from haptic stimulation in a frequency-dependent manner. The lower frequency $(1-2 \mathrm{~Hz})$ of haptic vibratory stimulation yielded the stronger feeling of animacy, while the higher frequency did not. The second study showed the importance of haptic stimulation in interpersonal communication. The presence of haptic communication in particular improved the quality of experience and the impression of the other person, even from a simple vibratory stimulation. The third study showed that haptic stimulation can modulate abstract feelings emerging in sensory modalities other than haptics.

Although all these studies have suggested the potential impact of haptic stimulation on abstract feelings, the empirical evidence of abstract feelings' emerging from haptic stimulation is much less than for the perceptual and sensory processes. The first reason may be that abstract feelings are too vague to examine empirically. Another reason relates to the difficulty of controlling haptic stimulation. However, at least with regard to the second reason, we have shown that simple vibratory haptic stimulation can be associated with abstract feelings. Therefore, it would be possible to investigate abstract feelings using simple haptic stimulation, which would accelerate the research concerning haptic stimulation associated with abstract feelings.

Future research directions regarding haptic stimulation and abstract feelings could include the following:

- Compare abstract feelings emerging from simple haptic stimulation and the real object.

- Examine the relation between haptic stimulation and abstract feelings other than animacy.

- Test factors other than frequency of haptic stimulation on abstract feelings.

- Measure the indices other than subjective evaluation, such as physiological responses to haptic stimulation.

- Relate controlled experimental studies to uncontrolled daily experience (e.g., patterns of frequency dependence for animacy and the frequencies of real-world animals).

All these studies could provide further insights into how humans conceive abstract feelings in response to haptic stimulation, and more generally, what abstract feelings emerge from haptic stimulation.

Finally, it would be worthwhile to state the potential applications of the results of the study of abstract feelings emerging from haptic stimulation. In the past decade, implementation of a sense of touch in communication devices has been advancing rapidly (e.g., humanoid and pet robots, mobile phones, and video games). Strong feelings of animacy might enhance the quality of communication through these devices. Furthermore, if other abstract feelings such as presence or intimacy were implemented in a future haptic device, the device should appreciate in value and would be expected to be widely used in real society. While the research discussed in this chapter is positioned as basic research on humans' cognitive process with regard to haptics, the results may help in designing and developing user-friendly devices by implementing the emergence of abstract feelings. 


\section{References}

Alais, D. \& Burr, D. (2004). The ventriloquist effect results from near-optimal bimodal integration, Current biology 14(3): 257-262.

Bertelson, P. \& de Gelder, B. (2005). Psychology of multimodal perception, in C. Spence \& J. Driver (eds), Crossmodal space and crossmodal attention, Oxford University Press, pp. 151-177.

Blake, R., Sobel, K. V. \& James, T. W. (2004). Neural synergy between kinetic vision and touch, Psychological science 15(6): 397-402.

Blakemore, S.-J., Boyer, P., Pachot-Clouard, M., Meltzoff, A., Segebarth, C. \& Decety, J. (2003). The detection of contingency and animacy from simple animations in the human brain, Cerebral cortex (New York, NY : 1991) 13(8): 837-844.

Bruno, N., Jacomuzzi, A., Bertamini, M. \& Meyer, G. (2007). A visual-haptic Necker cube reveals temporal constraints on intersensory merging during perceptual exploration, Neuropsychologia 45(3): 469-475.

Ernst, M. O. \& Banks, M. S. (2002). Humans integrate visual and haptic information in a statistically optimal fashion, Nature 415(6870): 429-433.

Ernst, M. O. \& Bülthoff, H. H. (2004). Merging the senses into a robust percept, Trends in cognitive sciences 8(4): 162-169.

Fujita, Y. \& Hashimoto, S. (1999). Experiments of haptic and tactile display for human telecommunication, 8th IEEE International Workshop on Robot and Human Interaction, pp. 334-337.

Fukuda, H. \& Ueda, K. (2010). Interaction with a Moving Object Affects One's Perception of Its Animacy, International Journal of Social Robotics 2(2): 187-193.

Gallese, V. \& Goldman, A. (1998). Mirror neurons and the simulation theory of mind-reading, Trends in cognitive sciences 2(12): 493-501.

Gao, T., McCarthy, G. \& Scholl, B. J. (2010). The wolfpack effect. Perception of animacy irresistibly influences interactive behavior., Psychological science 21(12): 1845-1853.

Gao, T., Newman, G. E. \& Scholl, B. J. (2009). The psychophysics of chasing: A case study in the perception of animacy, Cognitive psychology 59(2): 154-179.

Gao, T. \& Scholl, B. J. (2011). Chasing vs. stalking: Interrupting the perception of animacy., Journal of experimental psychology: Human perception and performance 37(3): 669-684.

Gibson, J. J. (1962). Observations on active touch., Psychological Review 69: 477-491.

Golding, J. \& Macleod, C. (1998). Intentional Forgetting: Interdisciplinary Approaches, Lawrence Erlbaum Assoc Inc.

Heider, F. \& Simmel, M. (1944). An Experimental Study of Apparent Behavior, The American Journal of Psychology 57(2): 243-259.

Katz, D. (1989). The World of Touch, Psychology Press.

Knapp, L. M. \& Hall, A. J. (1992). Nonverbal communication in human interaction, Holt Rinehart and Winston.

McGurk, H. \& MacDonald, J. (1976). Hearing lips and seeing voices, Nature 264(5588): 746-748.

Morito, Y., Tanabe, H. C., Kochiyama, T. \& Sadato, N. (2009). Neural representation of animacy in the early visual areas: a functional MRI study, Brain research bulletin 79(5): 271-280.

Morris, D. (1971). Intimate Behavior, 1st us edition edn, Triad Books.

Reiner, M. (2004). The role of haptics in immersive telecommunication environments, IEEE Transactions on Circuits and Systems for Video Technology 14(3): 392-401.

Richmond, V. P., McCroskey, J. C. \& Hickson, M. L. (2007). Nonverbal Behavior in Interpersonal Relations, 6 edn, Allyn \& Bacon. 
Rizzolatti, G. (2005). The mirror neuron system and its function in humans, Anatomy and embryology 210(5-6): 419-421.

Sallnäs, E.-L., Rassmus-Gröhn, K. \& Sjöström, C. (2000). Supporting presence in collaborative environments by haptic force feedback, ACM Transactions on Computer-Human Interaction 7(4): 461-476.

Santos, N. S., David, N., Bente, G. \& Vogeley, K. (2008). Parametric induction of animacy experience, Consciousness and cognition 17(2): 425-437.

Santos, N. S., Kuzmanovic, B., David, N., Rotarska-Jagiela, A., Eickhoff, S. B., Shah, J. N., Fink, G. R., Bente, G. \& Vogeley, K. (2010). Animated brain: a functional neuroimaging study on animacy experience, NeuroImage 53(1): 291-302.

Scholl, B. \& Tremoulet, P. (2000). Perceptual causality and animacy., Trends in cognitive sciences 4(8): 299-309.

Shams, L. \& Kim, R. (2010). Crossmodal influences on visual perception, Physics of life reviews 7(3): 269-284.

Shimojo, S. \& Shams, L. (2001). Sensory modalities are not separate modalities: plasticity and interactions, Current opinion in neurobiology 11(4): 505-509.

Takahashi, K., Mitsuhashi, H., Murata, K., Norieda, S. \& Watanabe, K. (2011a). Feelings of Animacy and Pleasantness from Tactile Stimulation: Effect of Stimulus Frequency and Stimulated Body Part, IEEE Int. Conf. on Systems, Man, and Cybernetics (SMC2011).

Takahashi, K., Mitsuhashi, H., Murata, K., Norieda, S. \& Watanabe, K. (2011b). Improving Shared Experiences by Haptic Telecommunication, International Conference on Biometrics and Kansei Engineering.

Takahashi, K., Mitsuhashi, H., Murata, K., Norieda, S. \& Watanabe, K. (2011c). Kansei Information and Factor Space of Nonverbal Interpersonal Communicaion - Psychological Investigation on Physical Contacts Modified by Japanese Onomatopoieas -, Transactions of Japan Society of Kansei Engineering 10(2): 261-268.

Takahashi, K., Mitsuhashi, H., Norieda, S., Murata, K. \& Watanabe, K. (2010). Frequency-dependence in haptic, visual, and auditory animacy perception, IEICE Human Communication Group Symposium 2010, pp. 331-336.

Takahashi, K., Mitsuhashi, H., Norieda, S., Sendoda, M., Murata, K. \& Watanabe, K. (2010). Japanese Onomatopoeias and Sound Symbolic Words in Describing Interpersonal Communication., The Proceedings of the International Conference on Kansei Engineering and Emotion Research 2010, pp. 2162-2171.

Tanaka, A., Koizumi, A., Imai, H., Hiramatsu, S., Hiramoto, E. \& de Gelder, B. (2010). I feel your voice. Cultural differences in the multisensory perception of emotion., Psychological science 21(9): 1259-1262.

Tremoulet, P. D. \& Feldman, J. (2000). Perception of animacy from the motion of a single object, Perception 29(8): 943-951.

Wang, D., Tuer, K. \& Ni, L. (2004). Conducting a real-time remote handshake with haptics, Proceedings of the 12th international conference on Haptic interfaces for virtual environment and teleoperator systems.

Zajonc, R. B. (1968). Attitudinal effects of mere exposure., Journal of personality and social psychology 9(2): 1-27. 


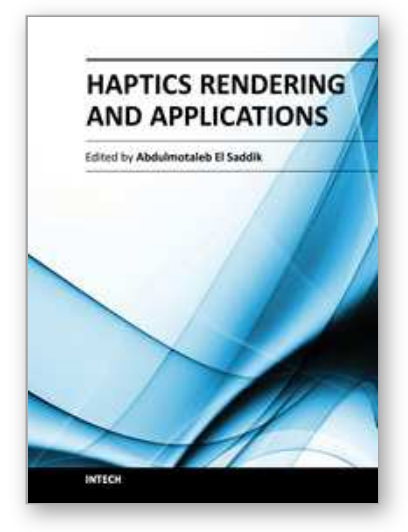

\author{
Haptics Rendering and Applications \\ Edited by Dr. Abdulmotaleb El Saddik
}

ISBN 978-953-307-897-7

Hard cover, 246 pages

Publisher InTech

Published online 27, January, 2012

Published in print edition January, 2012

There has been significant progress in haptic technologies but the incorporation of haptics into virtual environments is still in its infancy. A wide range of the new society's human activities including communication, education, art, entertainment, commerce and science would forever change if we learned how to capture, manipulate and reproduce haptic sensory stimuli that are nearly indistinguishable from reality. For the field to move forward, many commercial and technological barriers need to be overcome. By rendering how objects feel through haptic technology, we communicate information that might reflect a desire to speak a physicallybased language that has never been explored before. Due to constant improvement in haptics technology and increasing levels of research into and development of haptics-related algorithms, protocols and devices, there is a belief that haptics technology has a promising future.

\title{
How to reference
}

In order to correctly reference this scholarly work, feel free to copy and paste the following:

Kohske Takahashi, Hideo Mitsuhashi, Kazuhito Murata, Shin Norieda and KatsumiWatanabe (2012). Abstract Feelings Emerging from Haptic Stimulation, Haptics Rendering and Applications, Dr. Abdulmotaleb El Saddik (Ed.), ISBN: 978-953-307-897-7, InTech, Available from: http://www.intechopen.com/books/haptics-renderingand-applications/abstract-feelings-emerging-from-haptic-stimulation

\section{INTECH}

open science | open minds

\author{
InTech Europe \\ University Campus STeP Ri \\ Slavka Krautzeka 83/A \\ 51000 Rijeka, Croatia \\ Phone: +385 (51) 770447 \\ Fax: +385 (51) 686166 \\ www.intechopen.com
}

\author{
InTech China \\ Unit 405, Office Block, Hotel Equatorial Shanghai \\ No.65, Yan An Road (West), Shanghai, 200040, China \\ 中国上海市延安西路65号上海国际贵都大饭店办公楼 405 单元 \\ Phone: +86-21-62489820 \\ Fax: $+86-21-62489821$
}


(C) 2012 The Author(s). Licensee IntechOpen. This is an open access article distributed under the terms of the Creative Commons Attribution 3.0 License, which permits unrestricted use, distribution, and reproduction in any medium, provided the original work is properly cited. 